\title{
Particle acceleration and foreshock evolution in heliospheric shocks from self-consistent Monte Carlo simulations
}

\author{
A. Afanasiev ${ }^{* a}$, R. Vainio ${ }^{a}$, M. Battarbee ${ }^{a}$, M. Palmroth $^{b}$, S. von Alfthan ${ }^{b}$ and Y. \\ $\mathrm{Kempf}^{b}$ \\ ${ }^{a}$ Department of Physics and Astronomy, University of Turku, Turku, Finland \\ ${ }^{b}$ Earth Observation Unit, Finnish Meteorological Institute, Helsinki, Finland \\ E-mail: alexandr.afanasiev@utu.fi
}

\begin{abstract}
Self-consistent Monte Carlo simulations have been a fruitful approach to model particle acceleration dynamically coupled with the foreshock development in quasi-parallel shocks. There is the global Coronal Shock Acceleration (CSA) Monte Carlo simulation code that is capable of modelling self-consistent shock acceleration from the inner corona to the solar wind. However, in CSA, the resonant interactions of particles with the foreshock Alfvén waves are not modelled using the full resonance condition. The used simplified condition implies that particles of a given energy interact with only one particular spectral component of the wave spectrum. In contrast, the exact (within quasi-linear theory) treatment implies that such particles due to scattering in pitch angle can interact with different wave spectrum components. This changes the modelled particle acceleration efficiency of the shock and the wave spectrum evolution in the foreshock. We have developed a new self-consistent Monte Carlo simulation code, in which we overcome the previous simplification, and applied the two codes to model acceleration of protons in a parallel coronal shock. We also used the new code to simulate proton acceleration in an interplanetary shock. Due to the choice of the plasma and shock parameters, this simulation is applicable to quasi-parallel bow shock of the Earth. Comparison of the results shows that the resonant wave-particle interactions governed by the full resonance condition yield less efficient particle acceleration at the shock and the opposite energy dependence of the proton mean free path in the foreshock than the simplified treatment. Moreover, the Alfvén wave intensity spectrum resulting from the new code exhibits a $k^{-2}$ dependence at large wavenumbers, characteristic for both the coronal shock and the interplanetary one. This result is in agreement with that of a hybrid-Vlasov simulation (Vlasiator) of the foreshock evolution of the Earth's bow shock.
\end{abstract}

The 34th International Cosmic Ray Conference,

30 July- 6 August, 2015

The Hague, The Netherlands

* Speaker. 


\section{Introduction}

There is a variety of shock waves in the heliosphere. These include, e.g., planetary bow shocks formed by the persistent solar wind flow and travelling shocks driven by coronal mass ejections (CMEs) from the corona into the interplanetary space. Shocks represent one of the sources of energetic particles in the heliosphere. In particular, CME-driven shocks are responsible for particles observed in the so-called gradual solar energetic particle (SEP) events.

There are several mechanisms considered to accelerate particles in shocks. For a quasi-parallel shock, the particle acceleration mechanism is diffusive shock acceleration (DSA, e.g., [1]). In this mechanism, particles get energised by multiple crossings of the shock. An essential role in the mechanism is given to the Alfvénic turbulence in the vicinity of the shock, which scatters particles in pitch angle and facilitates the return of particles back to the shock. On the other hand, for efficient operation of DSA the turbulence in the foreshock region has to be much stronger than in the ambient corona/interplanetary space. This is achieved by means of the accelerated particles themselves, which generate Alfvén waves due to a streaming instability [1]. This couples the processes of particle acceleration and turbulence evolution.

Constructing a dynamical model of DSA, accounting for self-generated turbulence, is a challenging task, which has been attempted using numerical simulations. Self-consistent Monte Carlo simulations constitute one of the approaches, which has an advantage of providing detailed information on particle and turbulence distributions in the vicinity of the shock.

Current Monte Carlo simulations are built on quasi-linear theory [2] applicable for interactions of particles with weak slab-mode turbulence (represented by a spectrum of low-amplitude Alfvén waves). In quasi-linear theory, wave-particle interactions are resonant and governed by the following resonance condition (written in the wave rest frame):

$$
k_{\text {res }}=\frac{\Omega}{v \mu},
$$

where $k_{\text {res }}$ is the (resonant) wavenumber, $v$ and $\mu$ are the particle speed and pitch-angle cosine as measured in the wave rest frame, $\Omega=\gamma^{-1} \Omega_{0}, \Omega_{0}$ is the particle cyclotron frequency and $\gamma$ is the relativistic gamma factor. This resonance condition leads to anisotropic pitch-angle scattering of particles, for which the scattering rate is dependent on the pitch-angle. Moreover, it implies that in the scattering process a single particle may interact with different components of the wave spectrum.

During recent years, the Coronal Shock Acceleration (CSA) Monte Carlo code (see, e.g., [3] and [4]) has been extensively used to study particle acceleration and foreshock evolution in coronal shocks. The advantage of the code is that it allows for simulations of acceleration in parallel or oblique shocks on a global spatial scale (the shock can cover a distance of tens of solar radii in a single simulation). However, its performance is largely achieved by using a simplified resonance condition, $k_{\mathrm{res}}=\Omega / v$, which leads to isotropic pitch-angle scattering and implies that particles at a particular speed interact with only one particular spectral component of the wave intensity spectrum. The simplified treatment of the resonant interactions allows one to avoid the well-known problem of resonance gap in the quasi-linear theory. This, however, may lead to an over-efficiency of particle acceleration in CSA simulations. Also, the simplified treatment leaves out effects resulting from the ability of particles to interact with multiple wave spectral components. 
In this work, we study the effect of the full quasi-linear resonance condition of wave-particle interactions on particle acceleration and foreshock evolution, using our new Monte Carlo code named SOLar Particle Acceleration in Coronal Shocks (SOLPACS). Specifically, we simulate acceleration of protons in a parallel coronal shock, and compare SOLPACS results with those of CSA, obtained for the same initial and boundary conditions. We also present results from a SOLPACS simulation for a parallel interplanetary shock. Due to the choice of the plasma and shock parameters, those results, if considered in the shock frame, are applicable to quasi-parallel bow shock of the Earth. This gives us an opportunity to compare the SOLPACS results with those of Vlasiator, a hybrid-Vlasov simulation (kinetic ions, fluid electrons) code specifically designed for modelling the Earth's magnetosphere and its interaction with the solar wind [5].

\section{Simulation setup}

In both SOLPACS and CSA, energetic protons and Alfvén waves are traced along the mean magnetic field line (in a spatially 1-D simulation box) upstream of a shock. The version of CSA which we apply here is described in [3]. The numerical scheme implemented in SOLPACS to simulate wave-particle interactions is provided in [6]. The SOLPACS algorithm to model the injection of particles and their interaction with the shock is inherited from the applied version of CSA. Namely, the injected protons are characterised by a shock-frame velocity spectrum $d N_{\text {inj }} / d v=\left(N_{\text {inj }} / v_{1}\right) H\left(v-u_{1}\right) \mathrm{e}^{-\left(v-u_{1}\right) / v_{1}}$, where $N_{\text {inj }}=\varepsilon_{\text {inj }} n_{0} u_{1} t$ is the number of particles injected in time $t$ per unit cross-section of the magnetic flux tube, $n_{0}$ is the thermal proton density, $\varepsilon_{\mathrm{inj}}$ is the injection efficiency, $u_{1}$ is the upstream solar wind speed in the shock frame, $v_{1}$ is a parameter, and $H$ is the Heaviside step function. Instead of tracing particles in the shock's downstream, we employ a probability of return from the downstream region, which is determined by the scattering-centre compression ratio $r_{\mathrm{c}}$. We assume the plasma turbulence in the box to be due to outward-propagating (if considered in the solar wind frame) Alfvén waves, with the initial spectral form $\propto k^{-q_{0}}$, where $q_{0}$ is the spectral index. The initial level of turbulence is chosen to provide a prescribed value of the initial mean free path $\lambda_{0}$ for $100 \mathrm{keV}$ protons. As the wave-particle interaction effects are of the main concern here, we assume constant values of the thermal proton density $n_{0}$, magnetic field $B_{0}$ and solar wind speed $u_{0}$ in the whole simulation domain. For the coronal shock simulations we take $n_{0}=3.6 \times 10^{6} \mathrm{~cm}^{-3}, B_{0}=3.4 \times 10^{-5} \mathrm{~T}, u_{0}=12.4 \mathrm{~km} \mathrm{~s}^{-1}$, the simulation box length $L=1 R_{\odot}$, the shock speed $V_{\mathrm{s}}=1500 \mathrm{~km} \mathrm{~s}^{-1}, q_{0}=3 / 2$, and $\lambda_{0}=1 R_{\odot}$. Also, we fix the scattering-centre compression ratio to $r_{\mathrm{c}}=4$ and $v_{1}=375 \mathrm{~km} \mathrm{~s}^{-1}$. For the interplanetary shock simulation, $n_{0}=5 \mathrm{~cm}^{-3}, B_{0}=5 \times 10^{-9} \mathrm{~T}, u_{0}=400 \mathrm{~km} \mathrm{~s}^{-1}, L=0.1 \mathrm{AU}, V_{\mathrm{s}}=800 \mathrm{~km} \mathrm{~s}^{-1}$, $q_{0}=5 / 3, \lambda_{0}=0.1 \mathrm{AU}, r_{\mathrm{c}}=3.25$ and $v_{1}=200 \mathrm{~km} \mathrm{~s}^{-1}$. One can see that in the latter case the solar-wind speed in the shock frame, $u_{1}=400 \mathrm{~km} \mathrm{~s}^{-1}$.

Vlasiator is able to model the Earth's bow shock plasma environment in 6-D phase space (in 3-D ordinary space and 3-D velocity space), utilising electrons as MHD fluid and describing protons by their distribution function based on the Vlasov equation [5]. The simulation run employed in this study is 2-D in ordinary space (in the ecliptic plane). The interplanetary magnetic field $\mathbf{B}_{0}$ in the simulation is $5 \times 10^{-9} \mathrm{~T}$ in magnitude and is inclined by 5 degrees with respect to the solar wind velocity $\mathbf{u}_{0}$. Thus the shock is quasi-parallel at the sub-solar region. The solar wind speed 

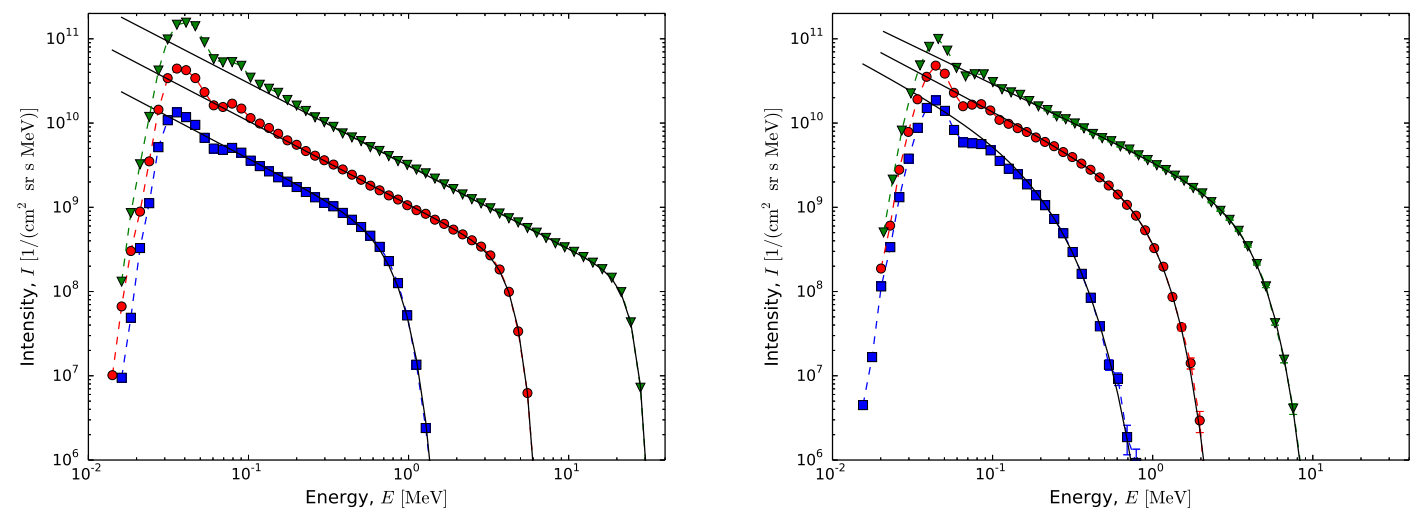

Figure 1: Particle energy spectra at the coronal shock, obtained with CSA (left panel) and SOLPACS (right panel) at $t=580 \mathrm{~s}$ for different values of the injection efficiency: $\varepsilon_{\text {inj }}=1.62 \times 10^{-6}$ (blue squares), $\varepsilon_{\text {inj }}=5.40 \times 10^{-6}$ (red circles) and $\varepsilon_{\text {inj }}=1.62 \times 10^{-5}$ (green triangles). The black curves represent fits to the spectral data. Note that the fitting was carried out at energies $E>0.2 \mathrm{MeV}$ in all cases.

$u_{0}=600 \mathrm{~km} \mathrm{~s}^{-1}$ and the proton density $n_{0}=3.3 \mathrm{~cm}^{-3}$. The velocity distribution function of solar wind protons is a Maxwellian with the temperature of $0.5 \mathrm{MK}$.

\section{Results}

\subsection{Coronal shock case}

For the case of a coronal shock, we have carried out simulations for three different particle injection efficiencies $\varepsilon_{\text {inj. }}$. The simulations were conducted until the time $t_{\max }=580 \mathrm{~s}$, which corresponds to the shock propagation distance of $1.25 R_{\odot}$. Figure 1 shows simulated proton energy spectra $I(E)$ at the shock, obtained at the end of simulation. Similar to [4], we fitted the resulting spectra by the function, $I(E)=C E^{-\beta} \exp \left\{-\left(E / E_{\mathrm{c}}\right)^{\delta}\right\}$, where $C, \beta, E_{\mathrm{c}}$, and $\delta$ are fitting parameters. The fits are shown in Fig. 1 as well. The best-fit values of the spectral parameters are given in Table 1. The spectra were fitted at $E>0.2 \mathrm{MeV}$. Note that when fitting the spectra corresponding to the weakest injection $\left(\varepsilon_{\text {inj }}=1.62 \times 10^{-6}\right)$, the power-law index $\beta$ was fixed to 1 (the theoretical value) as the power-law-like portion of the spectrum to be fitted was quite small (not recognisable at all in the case of the SOLPACS simulation).

Figure 2 shows simulated Alfvén wave spectra at the end $(t=580 \mathrm{~s})$ of the simulations for $\varepsilon_{\mathrm{inj}}=1.62 \times 10^{-5}$ in a close vicinity of the shock. In the SOLPACS spectrum the spectrum amplification extends up to the highest wavenumbers, exhibiting a $k^{-2}$ asymptotic form, whereas in the CSA spectrum the amplification drops off at $k \sim 10^{-3} \mathrm{~m}^{-1}$.

Figure 3, left panel shows spatial distributions of the particle mean free path $\lambda$ in the foreshock at different energies, calculated from CSA and SOLPACS data. One can see that the SOLPACS mean free path increases with energy whereas the CSA one shows the opposite dependence on energy. On the right panel of Fig. 3, the time evolution of the mean free path for $0.1 \mathrm{MeV}$ protons is presented. In CSA the mean free path achieves a steady state but in SOLPACS it does not. The behaviour of the mean free path at higher energies is qualitatively similar to the presented case. 

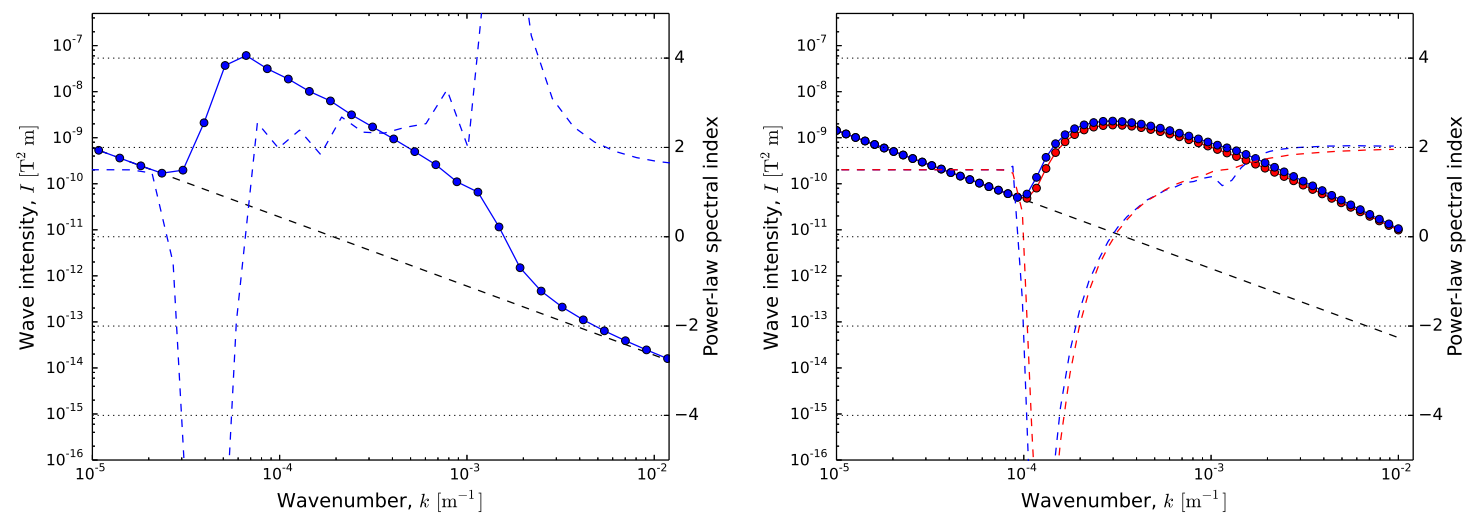

Figure 2: Alfvén wave intensity spectra obtained with CSA (left panel) and SOLPACS (right panel) for $\varepsilon_{\text {inj }}=1.62 \times 10^{-5}$ in the vicinity (upstream) of the coronal shock at $t=580 \mathrm{~s}$. The filled, blue and red, circles in the SOLPACS plot denote wave intensities corresponding to right- and left-handed circular polarizations, respectively. In both plots, the coloured dashed lines show spectral indices and the black dashed line shows the initial spectrum, normalised to provide $\lambda=1 R_{\odot}$ for $100 \mathrm{keV}$ protons.

\subsection{Interplanetary shock/Earth's bow shock case}

For the case of an interplanetary shock, we present a simulation for the injection efficiency $\varepsilon_{\text {inj }}=10^{-3}$. The total simulation time is $t_{\max }=18700 \mathrm{~s}$ ( $\sim 5$ hours $)$. Figure 4 , left panel shows a simulated particle energy spectrum at the shock at the end of the simulation, calculated in the fixed and shock frames of reference. One can recognise a power-law part in the spectra and a super-exponential cut-off, similar to the coronal shock case. However, the acceleration rate at the interplanetary shock is expectedly lower than at the coronal one. Figure 4, right panel shows a simulated Alfvén wave spectrum in the close vicinity of the shock at an earlier time, $t=3000 \mathrm{~s}$. Note the wavenumber dependence of the spectrum of right-handed waves being close to $\sim k^{-2}$.

Figure 5, left panel shows the distribution of transverse magnetic field components upstream of the quasi-parallel Earth's bow shock, obtained in the Vlasiator simulation at $t=500 \mathrm{~s}$ after the start of the run. Figure 5, right panel shows the power spectrum of magnetic fluctuations in the Vlasiator simulation in the vicinity of the shock. Note the $f^{-2}$ dependence of the spectrum obtained.

\begin{tabular}{lccccc}
\hline $\begin{array}{l}\text { Simulation } \\
\text { type }\end{array}$ & $\varepsilon_{\text {inj }} \times 10^{6}$ & $\beta$ & $\begin{array}{c}E_{\mathrm{c}} \\
(\mathrm{MeV})\end{array}$ & $\delta$ & $\begin{array}{c}C \times 10^{-9} \\
\left(\mathrm{pfu} \mathrm{MeV}^{\beta}\right)\end{array}$ \\
\hline \multirow{3}{*}{ CSA } & 1.62 & 1 (fixed) & 0.78 & 3.1 & 0.38 \\
& 5.40 & 0.99 & 4.3 & 4.8 & 1.1 \\
& 16.2 & 0.98 & 24. & 6.3 & 3.1 \\
\hline \multirow{3}{*}{ SOLPACS } & 1.62 & 1 (fixed) & 0.18 & 1.4 & 0.80 \\
& 5.40 & 1.0 & 0.89 & 2.2 & 1.4 \\
& 16.2 & 0.92 & 3.8 & 2.4 & 3.5 \\
\hline
\end{tabular}

Table 1: Best-fit values of the fitting function parameters. The pfu unit stands for the particle flux unit $\left(1 \mathrm{pfu}=1 \mathrm{~cm}^{-2} \mathrm{sr}^{-1} \mathrm{~s}^{-1} \mathrm{MeV}^{-1}\right)$. 

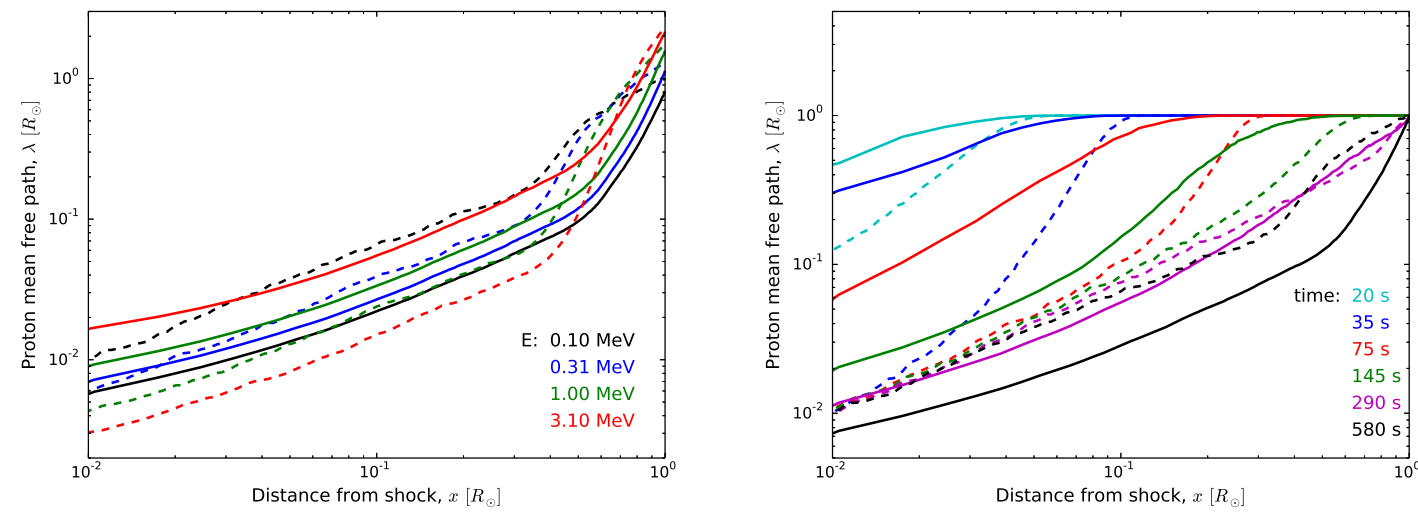

Figure 3: Left panel: Proton mean free path as a function of distance from the coronal shock at different energies, calculated from the SOLPACS (continuous curves) and CSA data (dashed curves) corresponding to $\varepsilon_{\text {inj }}=1.62 \times 10^{-5}$ and $t=580 \mathrm{~s}$. Right panel: Time evolution of the proton mean free path in the foreshock at $E=0.1 \mathrm{MeV}$ in SOLPACS (continuous curves) and in CSA (dashed curves) for $\varepsilon_{\text {inj }}=1.62 \times 10^{-5}$.
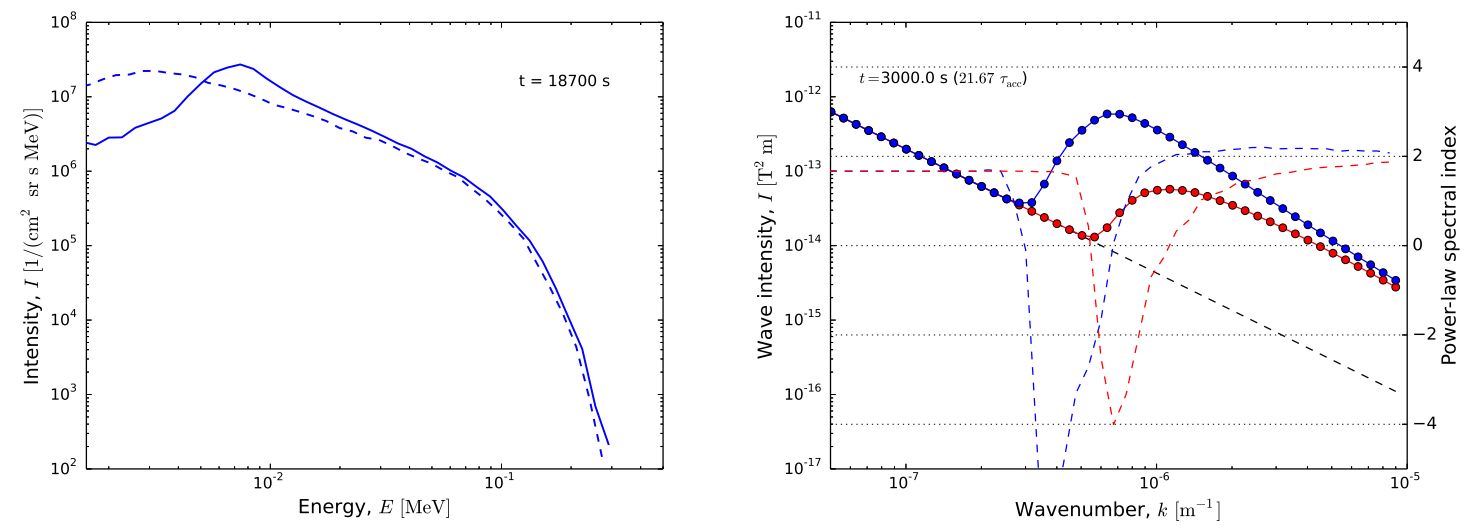

Figure 4: Left panel: Particle energy spectrum at the interplanetary shock, obtained with SOLPACS at $t=18700 \mathrm{~s}$ for the injection efficiency $\varepsilon_{\text {inj }}=10^{-3}$, as measured in the fixed frame (continuous curve) and in the shock frame (dashed curve). Right panel: Alfvén wave intensity spectrum in the vicinity of the interplanetary shock, obtained with SOLPACS for the injection efficiency $\varepsilon_{\text {inj }}=10^{-3}$ at $t=3000 \mathrm{~s}$.

\section{Discussion}

The spectra presented in Fig. 1 clearly demonstrate that the wave-particle interactions controlled by the full quasi-linear resonance condition yield less efficient acceleration of particles (in terms of the cut-off energy $E_{\mathrm{c}}$ ) at a shock than those determined by the simplified resonance condition.

The distinctions between the wave spectra resulting from CSA and SOLPACS, shown in Fig. 2 , are due to the different forms of the applied resonance condition as well. In Bell's steadystate theory of DSA the wave spectrum $I_{\mathrm{W}}(k)$ has a break at $k=k_{\mathrm{b}}$, so that $I_{\mathrm{W}}(k) \propto k^{\sigma-6}$, where $\sigma=3 r_{\mathrm{c}} /\left(r_{\mathrm{c}}-1\right)$, at $k \ll k_{\mathrm{b}}$, and $I_{\mathrm{W}}(k) \propto k^{-3}$ at $k \gg k_{\mathrm{b}}$ (note also that in Bell's theory the spectrum has an abrupt cut-off at $k_{0}=\Omega / v_{0}$, where $v_{0}$ is the injected particle beam velocity in the wave 

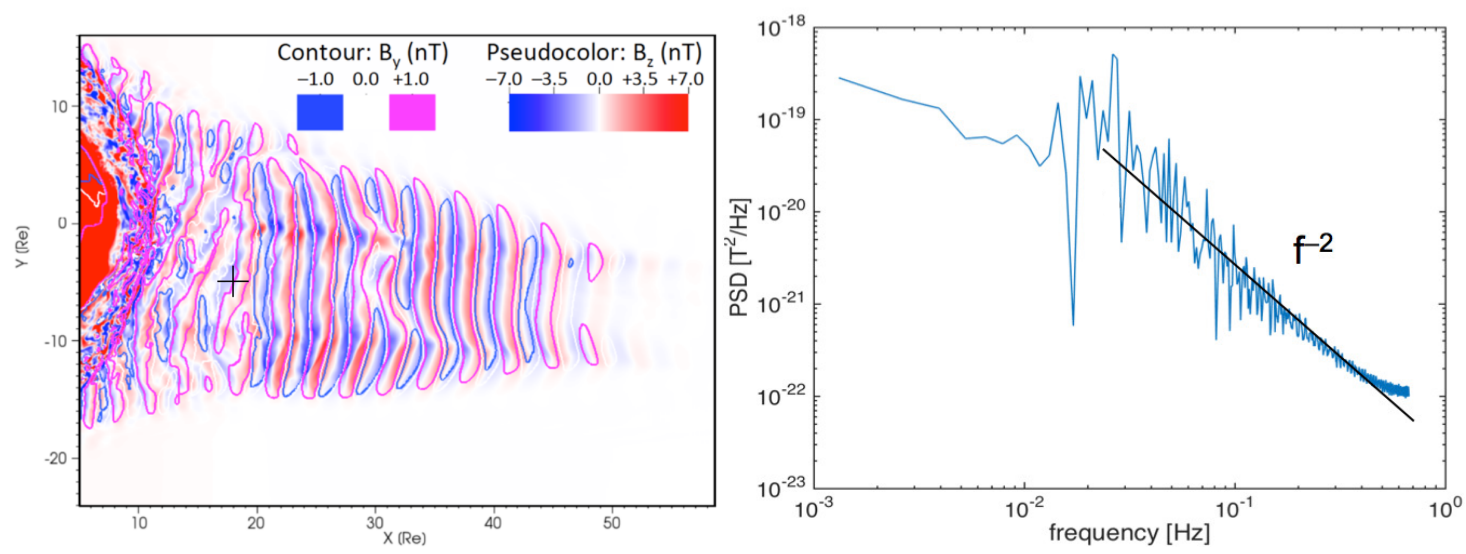

Figure 5: Left panel: Distribution of transverse magnetic field components upstream of the quasi-parallel Earth's bow shock, obtained with Vlasiator at $t=500 \mathrm{~s}$ after the start of the run. The "+" sign marks the point $(x, y)=(18,-5) R_{\mathrm{e}}$, where the power spectrum of magnetic fluctuations (shown on the right panel) was obtained. Right panel: Power spectrum of magnetic field fluctuations.

frame). For the spectra presented in Fig. 2, $\sigma=4, k_{\mathrm{b}}=4.2 \times 10^{-4} \mathrm{~m}^{-1}$ and $k_{0}=10^{-3} \mathrm{~m}^{-1}$. One can see that the spectral index of the CSA spectrum increases in the range between 2 and 3 in the wavenumber interval of $10^{-4} \lesssim k \lesssim 10^{-3} \mathrm{~m}^{-1}$ (where the bulk of particles is in resonance) in agreement with Bell's theory. The SOLPACS spectrum exhibits a variable spectral index $q \lesssim$ 2 at $k \leq k_{0}$, thus contradicting Bell's steady-state theory, and takes an asymptotic form $\sim k^{-2}$. SOLPACS simulations show that the $k^{-2}$ asymptotic form of the wave spectrum is characteristic for both the foreshock of a coronal shock and that of an interplanetary shock. Moreover, this result is supported by the Vlasiator simulation.

The form of the wave spectrum obtained from SOLPACS provides an increase in the foreshock particle mean free path $\lambda$ with energy, shown in Fig. 3. This is again in contradiction with the steady-state theory, where $\lambda \propto E^{-1 / 2}$ (in the non-relativistic regime) for the chosen values of the shock parameters. The mean free path decreasing with time in SOLPACS is a clear evidence of the effect of high-energy particles on the scattering conditions of low-energy ones in the foreshock, which is missing from the steady-state theory and CSA. The high-energy particles generate Alfvén waves that interact with low-energy particles, enhancing their pitch-angle scattering and return back to the shock. The persistent increase of the wave energy at the wavenumbers resonant with low-energy particles leads to the reduction of the particle mean free path close to the shock during the whole simulation. This is in contrast to the picture provided by the steady-state theory and CSA, in which particles are bound to particular wavenumbers and the mean free path reaches the steady state.

The wave spectrum in Fig. 4 and the Vlasiator results in Fig. 5 correspond approximately to the same dimensionless time $t^{*}=t / \tau_{\text {acc }}$, where $\tau_{\text {acc }}$ is the characteristic acceleration time in the system. The fluctuations in the $B_{y}$ and $B_{z}$ shown on the left panel of Fig. 5 have about 90 degrees phase shift consistent with elliptic polarization and, when analysed more closely, appear to be predominantly right-handed in the plasma frame, as in the quasi-linear simulation (Fig. 4). 


\section{Summary}

We have presented Monte Carlo simulations of diffusive shock acceleration of protons interacting self-consistently with Alfvén waves in the upstream region of a parallel heliospheric (coronal and interplanetary) shock. In the latter case, we have made comparisons of some properties of the waves with those resulting from the Vlasiator simulation model for the Earth's bow shock. We have found that, in comparison with the simplified treatment, resonant wave-particle interactions controlled by the full quasi-linear resonance condition, yield: $a$ ) Less efficient particle acceleration at the shock. b) $k^{-2}$ asymptotic form of the wave spectrum, which is in agreement with the Vlasiator simulation result. $c$ ) Increasing particle mean free path as a function of energy, in contrast to the steady-state theory.

\section{Acknowledgments}

This project has received funding from the European Union's Horizon 2020 research and innovation programme under grant agreement No 637324 (HESPERIA) and from the Academy of Finland (grant numbers 258963, 267186, 138599 and 267144). The Monte Carlo simulations were partly performed using the Finnish Grid Infrastructure (FGI) project (Turku, Finland). Vlasiator (http://vlasiator.fmi.fi) was developed with the European Research Council Starting grant (200141QuESpace) granted to MP in 2007. The Academy of Finland has also supported the Vlasiator development. We gratefully acknowledge CSC-IT Center for Science for granting us pilot usage of the Sisu supercomputer. VisIt [7] is used to visualise Vlasiator data.

\section{References}

[1] A. R. Bell, The acceleration of cosmic rays in shock fronts. I, MNRAS 182 (1978) 147-156.

[2] J. R. Jokipii, Cosmic-Ray Propagation. I. Charged Particles in a Random Magnetic Field, ApJ 146 (1966) 480.

[3] R. Vainio and T. Laitinen, Monte Carlo Simulations of Coronal Diffusive Shock Acceleration in Self-generated Turbulence, ApJ 658 (2007) 622-630.

[4] R. Vainio, A. Pönni, M. Battarbee, H. E. J. Koskinen, A. Afanasiev, and T. Laitinen, A semi-analytical foreshock model for energetic storm particle events inside 1 AU, J. Space Weather Space Clim. 4 (2014) A8.

[5] S. von Alfthan, D. Pokhotelov, Y. Kempf, S. Hoilijoki, I. Honkonen, A. Sandroos, and M. Palmroth, Vlasiator: First global hybrid-Vlasov simulations of Earth's foreshock and magnetosheath, J. Atmospheric Solar-Terrestrial Phys. 120 (2014) 24-35.

[6] A. Afanasiev and R. Vainio, Monte Carlo Simulation Model of Energetic Proton Transport through Self-generated Alfvén Waves, ApJS 207 (2013) 29.

[7] H. Childs, E. Brugger, B. Whitlock, J. Meredith, S. Ahern, D. Pugmire, K. Biagas, M. Miller, C. Harrison, G. H. Weber, H. Krishnan, T. Fogal, A. Sanderson, C. Garth, E. Wes Bethel, D. Camp, O. Rübel, M. Durant, J. M. Favre, and P. Navrátil, VisIt: An End-User Tool For Visualizing and Analyzing Very Large Data, in High Performance Visualization-Enabling Extreme-Scale Scientific Insight (2012), 357-372. 